\title{
Editorial
}

Digestion

\section{Lactose Intolerance: New Insights due to Blinded Testing?}

\author{
Benjamin Misselwitz \\ Division of Gastroenterology and Hepatology, University Hospital Zurich, Zurich, Switzerland
}

All human newborns and basically all newborn mammals express the enzyme lactase in the small intestine. This enables the digestion of lactose and the ability to thrive on milk as the only nutrient. However, later in life lactase expression is downregulated in animals and most humans, a process which possibly facilitates weaning. After lactase downregulation, lactose will reach the colonic microbiota, resulting in the production of short-chain fatty acids, hydrogen and methane, which can potentially cause gastrointestinal symptoms.

$5,000-10,000$ years BC in central Europe, a mutation within the promoter of the lactase gene occurred, resulting in the persistence of lactase expression also in adults [1]. This LCT-13910C>T mutation has been of tremendous evolutionary advantage, increasing evolutionary fitness by up to $19 \%$ in each generation [2]. Lactase persistence enabled our farming ancestors to ingest milk in large quantities, providing a clean source of liquid, calories and vitamin $\mathrm{D}$. Due to these benefits, the mutation in the lactase promoter spread and in central and northern Europe as well as in North America lactase persistence (resulting in lactose digestion) is now the most frequent phenotype. However, in most areas of the world, including most parts of Asia and Africa, lactase nonpersistence and lactose maldigestion (LM) remain the most frequent conditions. For European and North American physicians, it is thus important to realize that lactase nonpersistence and LM are not a disease but a normal human phenotype [3].

\section{KARGER}

(c) 2014 S. Karger AG, Base

0012-2823/14/0901-0072\$39.50/0

E-Mail karger@karger.com

www.karger.com/dig
Lactose intolerance (LI) refers to the development of symptoms after ingestion of lactose. LI can explain symptoms for some patients with diarrhea and abdominal pain. Learning from experience, patients will decrease milk and calcium consumption, possibly resulting in an increased risk for osteoporosis and bone fractures [4].

LI is not synonymous with and should not be confused with LM. Symptom development depends on the amount of lactose ingested, the colonic microbiota, individual sensitivity and subjective expectations [5]. Patient history can therefore be misleading: when tested in a blinded setting, individuals with self-diagnosed severe LI were able to ingest up to $12 \mathrm{~g}$ of lactose (corresponding to $250 \mathrm{ml}$ of milk) and some of these individuals did not even have the LM phenotype [6].

A 2010 NIH conference therefore proposed a new definition of LI [7]. LI now refers to the development of symptoms after blinded lactose challenge in an individual with $L M$. Strictly speaking, testing of LI would therefore be a two-step procedure. First, the LM phenotype should be established. For this task, many tools including genetic analysis, the $\mathrm{H}_{2}$-breath test and measurements of lactase expression in duodenal biopsies are available. For the assessment of LI, blinded testing would be required, but so far no validated clinical tools are available. Therefore, LI according to the NIH definition cannot be diagnosed in today's clinical practice.

In the current issue of Digestion, Latorre et al. [8] report the first study where testing for LI has been performed according to the NIH definition. 121 healthy 
Chilean students without gastrointestinal symptoms ingested $25 \mathrm{~g}$ of lactose or sucrose in a double-blinded setting, and $\mathrm{H}_{2}$-concentration in expiratory air and symptoms were monitored. Among the study subjects, 69 out of 121 individuals (57\%) had genetic lactase nonpersistence and 60 of those $69(87 \%)$ had a positive $\mathrm{H}_{2}$-breath test. The correlation of the genetic test and the $\mathrm{H}_{2}$-breath test is therefore not perfect. Reasons for discrepancies include a colon flora unable to produce hydrogen ('non- $\mathrm{H}_{2}$ producers'), secondary lactase deficiency and complex genetic mechanisms determining lactase expression which are not entirely understood yet.

Importantly, $56 \%$ of all the subjects with proven lactose malabsorption in the $\mathrm{H}_{2}$-breath test also had LI. The threshold chosen for scoring 'positive' symptoms in this study was relatively low (6-point difference relative to placebo on a 50-point scale) and a more stringent threshold might have decreased the percentage of individuals with true LI according to the NIH definition. In any case, a test distinguishing approximately $50 \%$ of individuals potentially benefitting from further treatment (lactose-reduced diet or lactase enzyme treatment) could be clinically very useful.

Should blinded testing now be universally introduced into clinical practice? Not necessarily. These results just reflect the situation in a population of healthy young students (mean age 21 years). In contrast, the patient population referred to LI testing likely has a higher prevalence of irritable bowel syndrome (IBS), self-diagnosis of LI and/or psychiatric comorbidity.

Furthermore, future studies need to address whether blinded testing can actually improve patient management. It would be interesting to learn whether blinded testing would improve calcium intake and bowel symptoms several months after lactose tolerance testing. Blinded multi- ple-dose testing to determine the maximum tolerated lactose dosage [9] might further improve dietary management. Therefore, assessment of symptoms does not need to be done simultaneously to an $\mathrm{H}_{2}$-breath test, but could also be done at patients' homes in an inexpensive manner.

Finally, it seems appropriate to regard LI not as an isolated condition, but as a manifestation of functional bowel disorders including IBS. In fact, in Chinese probands with a prevalence of LM approximating $100 \%$, symptoms after lactose challenge occurred at a much higher frequency in individuals fulfilling diagnostic criteria of IBS compared to controls [9]. However, lactose is not the only nutrient fermented by the colonic microbiota after maldigestion in the small intestine, and a group of nutrients with a similar profile (FODMAPs, i.e. fermentable oligo-, di- and monosaccharides and polyols) has been identified. A diet low in FODMAPs has recently been shown to improve symptoms in patients with IBS [10]. Lactose could be the leading FODMAP in IBS patients with LM and a lactose-rich diet. For other IBS patients, other nutrients could be causing symptoms. Therefore, rational dietary management of IBS patients might first include a lactulose test meal in a controlled and blinded setting [11] (lactulose will be fermented by the colon microbiota and thus act as a FODMAP in all individuals). Only individuals with symptoms should be referred for more specialized testing including LM and blinded LI testing.

Taken together, blinded testing of nutrients is a new concept in functional diagnostics in gastroenterology with the potential to improve the management of patients with nutrition-dependent abdominal symptoms. However, further tests will be needed to determine the application of LI testing which will benefit our patients best.

\section{References}

$>1$ Enattah NS, et al: Identification of a variant associated with adult-type hypolactasia. Nat Genet 2002;30:233-237.

$>2$ Bersaglieri $\mathrm{T}$, et al: Genetic signatures of strong recent positive selection at the lactase gene. Am J Hum Genet 2004;74:1111-1120.

>3 Ingram CJ, Mulcare CA, Itan Y, Thomas MG, Swallow DM: Lactose digestion and the evolutionary genetics of lactase persistence. Hum Genet 2009;124;579-591.

$\checkmark 4$ Wilt TJ, et al: Lactose Intolerance and Health Evidence Report/Technology Assessment. Rockville, Agency for Healthcare Research and Quality, 2010.
5 Misselwitz B, et al: Lactose malabsorption and intolerance: pathogenesis, diagnosis and treatment. United European Gastroenterol J 2013;1:151-159.

-6 Suarez FL, Savaiano DA, Levitt MD: A comparison of symptoms after the consumption of milk or lactose-hydrolyzed milk by people with self-reported severe lactose intolerance. N Engl J Med 1995;333:1-4.

7 Suchy FJ, et al: NIH consensus development conference statement: lactose intolerance and health. NIH Consens State Sci Statements 2010;27:1-27.

$>8$ Latorre G, et al.: Prevalence of lactose intolerance in Chile: a double-blind placebo study. Digestion 2014;90:18-26.
9 Yang J, et al: Prevalence and presentation of lactose intolerance and effects on dairy product intake in healthy subjects and patients with irritable bowel syndrome. Clin Gastroenterol Hepatol 2013;11:262-268.e1.

10 Halmos EP, Power VA, Shepherd SJ, Gibson PR, Muir JG: A diet low in FODMAPs reduces symptoms of irritable bowel syndrome. Gastroenterology 2014;146:67.

11 Le Neve B, et al: A combined nutrient and lactulose challenge test allows symptombased clustering of patients with irritable bowel syndrome. Am J Gastroenterol 2013; $108 ; 786-795$ 